\title{
Health-care associated infections. Patient characteristics and influence on the clinical outcome of patients admitted to icu. envin-helics registry data
}

X Nuvials ${ }^{1 *}$, M Palomar $^{2}$, F Alvarez-Lerma ${ }^{3}$, P Olaechea ${ }^{4}$, S Otero ${ }^{5}$, S Uriona ${ }^{5}$, M Catalán ${ }^{6}$, R Gimeno ${ }^{7}$, MP Gracia ${ }^{3}$, I Seijas ${ }^{8}$, ENVIN-HELICS

From ESICM LIVES 2015

Berlin, Germany. 3-7 October 2015

\section{Introduction}

Health care associated infections (HCAI) are frequent in patients admitted to ICU and have a great impact on the clinical outcome.

\section{Objectives}

To analyze the characteristics of patients developing HCAI during ICU admission and their influence on the clinical outcome.

\section{Methods}

Prospective, observational, multicenter and voluntary enrollment study (Spanish registry ENVIN-HELICS) [1]. All patients admitted to ICU for $>24$ hours between 1st April and 30th June during the period from 2006 to 2013 were included. All episodes of HCAI were recorded during the follow-up. HCAI were categorized as Ventilator Associated Neumonia (VAP), Primary bacteriemia and Catheter related bacteriemia (PB-RCB) and catheter-associated Urinary Tract Infection (CAUTI)

Table 1

\begin{tabular}{|c|c|c|c|c|}
\hline & No infection $(n=89.838)$ & non HCAI $(n=27.850)$ & HCAI $(n=11.349)$ & $\mathbf{p}$ \\
\hline Age: average \pm SD & $62.5 \pm 16.4$ & $62.6 \pm 16.3$ & $60.9 \pm 16.3$ & $P<0.05$ \\
\hline Sex: n(\%)male Female & 58,979 (65.6) 30,850 (34.4) & 18,115 (65.0) 9,735 (35.0) & $7,2928(65.2) 3,956(34.8)$ & NS \\
\hline APACHE II: average \pm SD & $12.53 \pm 7.5$ & $18.6 \pm 8.2$ & $19.6 \pm 8.0$ & $P<0.05$ \\
\hline $\begin{array}{l}\text { Type of admission: } \mathrm{n}(\%) \text { Coronary Medical } \\
\text { Elective surgery Emergency surgery } \\
\text { Trauma }\end{array}$ & $\begin{array}{l}27,363(92.5) 29,506(55.4) \\
20,268(87.6) 5,467(43.5) 6,146 \\
(67.6)\end{array}$ & $\begin{array}{l}1,417(4.8) 17,204(32.3) \\
1,859(8.0) 5,705(45.5) 1,395 \\
(15.4)\end{array}$ & $\begin{array}{l}793(2.7) 6,514(12,2) 1,004 \\
(4.3) 1,387(11.0) 1,546 \\
(17.0)\end{array}$ & $P<0.05$ \\
\hline $\begin{array}{l}\text { Antimicrobial treatment at admission } \\
(\mathrm{n}=28.343): \mathrm{n}(\%)\end{array}$ & $10,878(38.4)$ & $13,228(46.7)$ & $4,237(14.9)$ & $P<0.05$ \\
\hline $\begin{array}{l}\text { Extrarenal depuration technic }(n=6.400) \text { : } \\
n(\%)\end{array}$ & $2,163(33.8)$ & $2,509(39.2)$ & $1,728(27.0)$ & $P<0.05$ \\
\hline Parenteral Nutrition ( $n=14.565): \mathrm{n}(\%)$ & $5,064(34.8)$ & $6,069(41.7)$ & $3,432(23.6)$ & $P<0.05$ \\
\hline Mortality: n (\%) & $5,764(6,4)$ & 4,966 (17.8) & $2,976(26.2)$ & $P<0.05$ \\
\hline Length of stay: days \pm SD & $4.9 \pm 4.8$ & $10.1 \pm 9.4$ & $22.8 \pm 16.2$ & $P<0.05$ \\
\hline
\end{tabular}

${ }^{1}$ Hospital Universitari Arnau de Vilanova, IRB Lleida, Lleida, Spain

Full list of author information is available at the end of the article 
according the HELICS definitions [2]. Patients were categorized as: no infection (NI) when they had not infection during admission, no-HCAI when they had infection not related with health-care during admission, and HCAI when they had at least 1 episode of HCAI. Demographic data, risk factors, length of stay and mortality were recorded. Univariate analysis was done using Chi-square test. $\mathrm{p}$ value $<0,05$ was considered statistical significant.

\section{Results}

Among 129,037 patients admitted to ICU, 58,706 infections were recorded, of whom 15,490 (26.4\%) were HCAI: 6,068 (10.3\%) VAP, 4,943 (8.4\%) CAUTI and 4,479 (7.6\%) PB-CRB. 12,612 (82.1\%) of HCAI were ICU acquired, 1,966 (12,7\%) were hospital acquired and 686 $(4,4 \%)$ were acquired in the community. Table 1 shows demographic data, risk factors and the clinical outcome of patients accordingly the infectious status.

\section{Conclusions}

Patients admitted to ICU with HCAI have a worse clinical outcome( higher mortality and length of stay), and are more severely ill on admission than patients with non related HCAI.

\section{Authors' details}

${ }^{1}$ Hospital Universitari Arnau de Vilanova, IRB Lleida, Lleida, Spain. ${ }^{2}$ Hospital Universitari Arnau de Vilanova. IRB Lleida, Intensive Care Medicine, Lleida, Spain. ${ }^{3}$ Hospital del Mar, Intensive Care Medicine, Barcelona, Spain. ${ }^{4}$ Hospital de Galdakao, Intensive Care Medicine, Galdakao, Spain. ${ }^{5}$ Hospital Universitari Vall d'Hebron, Medicina Preventiva, Barcelona, Spain. ${ }^{6}$ Hospital 12 de Octubre, Intensive Care Medicine, Mardrid, Spain. ${ }^{7}$ Hospital Univesitario La $\mathrm{Fe}$, Intensive Care Medicine, Valencia, Spain. ${ }^{8}$ Hospital de Cruces, Intensive Care Medicine, Baracaldo, Spain.

Published: 1 October 2015

\section{References}

1. [http://hws.vhebron.net/envin-helics/]

2. Hospital in Europe Link for Infection Control through Surveillance (HELICS). Version 6.1. Sep. 2004. Surveillance of Nosocomial Infections in Intensive Care Units. , En: http://www.ecdc.europa.eu/IPSE/protocols/ icu_protocol.pdf.

doi:10.1186/2197-425X-3-S1-A82

Cite this article as: Nuvials et al:: Health-care associated infections.

Patient characteristics and influence on the clinical outcome of patients admitted to icu. envin-helics registry data. Intensive Care Medicine Experimental 2015 3(Suppl 1):A82.

\section{Submit your manuscript to a SpringerOpen ${ }^{\mathcal{D}}$ journal and benefit from:}

- Convenient online submission

- Rigorous peer review

- Immediate publication on acceptance

- Open access: articles freely available online

- High visibility within the field

- Retaining the copyright to your article

Submit your next manuscript at $>$ springeropen.com 